\title{
Effects of stimulus similarity on perceptual and conceptual comparisons
}

\author{
DONALD W. ROBBINS and L. E. BOURNE, JR. \\ University of Colorado, Boulder, Colorado
}

\begin{abstract}
Subjects were to judge as rapidly as possible whether pairs of geometric designs were the same or different on a given relevant dimension (size, shape, or color). Response times varied depending on the number of values on nonrelevant dimensions shared by the comparison stimuli (stimulus similarity). "Same" responses were facilitated by stimulus similarity, regardless of whether the stimuli were perceptually available for comparison or available (identified by verbal labels) only for conceptual or memorial comparison. "Different" responses were slowed by similarity in the perceptual task, but facilitated in the conceptual task. An experimental manipulation that permitted the subjects to identify the comparison stimuli prior to a memorial comparison did not change the basic similarity function. The data were generally more consistent with an activation rather than with a search/retrieval theory of short-term memory. A descriptive linear model with two similarity-dependent parameters for response competition and semantic distance effects gives a close account of the data.
\end{abstract}

The purpose of these experiments was to examine the effects of stimulus similarity on the speed and accuracy of stimulus comparison. The task for the subjects was to decide whether two stimuli were the same or different on a designated dimension, such as color. The similarity of the two stimuli was varied by manipulating the number of other dimensions on which they were identical or different. We were particularly concerned with the difference that stimulus similarity might make in judging sameness or difference when the stimuli were perceptually available for comparison or available for comparison only in memory. This difference has important implications for models of memory and for decisions about which processes in memory are similarity dependent.

The question we deal with is not new. There have, in fact, been many studies of similarity effects in stimulus comparisons. In the case of perceptual comparisons, the common finding is that mismatches on irrelevant dimensions (dissimilarity between stimuli) inhibit or slow down "same" responses. That is, if two stimuli are identical on the dimension of comparison, the correct response will be inhibited by increasing the number of differences on irrelevant dimensions. This effect has been shown over a variety of conditions and types of stimuli and is not, at this time, an issue of dispute (Egeth, 1966; Hawkins \& Shigley, 1972; Miller \&

This research was conducted within the Institute of Cognitive Science and is Publication No. 102 of the Institute. The work was supported by Research Grant BNS 76-81416 from the National Science Foundation. The author's mailing address is: Institute of Cognitive Science, University of Colorado, Boulder, Colorado 80309.
Bauer, 1981). The effect of stimulus similarity on "different" responses, however, is inconsistent, sometimes increasing reaction time (RT) (Connor, 1972; Williams, 1974) but often having no effect (Krueger, 1973; Well \& Green, 1972) in the empirical studies that are presently available.

The usual explanation for interdimensional similarity effects in the perceptual comparison findings entails the operation of two underlying processes, response competition and attention distraction. Response competition operates such that differences on irrelevant dimensions bias the subject to say "different." Thus, increasing the number of differences on irrelevant dimensions slows down "same" responses and speeds up "different" responses. The distraction process operates such that the more differences on irrelevant dimensions, the more likely is the subject's attention to be attracted to those dimensions. Thus, the more mismatches, the slower RT should be regardless of whether the correct response is "same" or "different." Therefore, "same" responses should be inhibited by the response competition and distraction process, both of which increase in potency with the number of mismatches on irrelevant dimensions. Response competition and distraction have antagonistic effects on "different" responses. If response competition effects are stronger than distraction effects, "different" responses should be enhanced by increasing the number of mismatched irrelevant dimensions. Just the opposite result should obtain if distraction effects are stronger than response competition effects. Obviously, if the two effects are equal, the number of irrelevant dimensions will have no observable influence. 
The literature on memorial or conceptual comparisons is not quite as systematic as, and is not easily compared with, the literature on perceptual comparisons. In tasks in which the subject is required to judge whether two stimuli belong to the same category, similarity has been shown to enhance "same" responses and to degrade "different" responses (Caramazza, Hersh, \& Torgerson, 1976), much in the manner of perceptual comparisons. These studies are based typically on the rated similarity of members of natural categories rather than on manipulations of features of the stimuli. To our knowledge, there are no relevant experiments on interdimensional similarity effects. Studies of the symbolic distance effect are also pertinent. In these experiments, subjects are taught labels for a set of stimuli that vary exclusively along some single dimension, such as size. The labels are then presented in pairs, and the subject must make a judgment about which of the two referents has a more extreme value on the underlying dimension, essentially a "difference" response. Typically, it has been found that speed of response is directly related to distance between stimuli (i.e., dissimilarity) on the underlying continuum (Kosslyn, Murphy, Bemesderfer, \& Feinstein, 1977; Moyer \& Bayer, 1976). Thus, intradimensional similarity tends to inhibit the "different" judgment here just as interdimensional similarity does in other memorial and some perceptual tasks.

While there is considerable literature dealing with similarity effects on "same"-"different" judgments, only a study by Tversky (1969), to our knowledge, included both conceptual and perceptual stimuli. The focus of that study, in which subjects judged whether two stimuli either were identical or referred to identical percepts, was on the ability of subjects to adopt either a verbal or an imagistic form of stimulus representation, depending on the frequency with which pictures or words were used as stimuli. In contrast, the present study aimed to determine whether the effects of similarity on "same" vs. "different" judgment times are parallel for conceptual and perceptual stimuli.

In all conditions of the present experiments, comparisons were made between two geometrical designs that could differ on as many as three dimensions: size, color, and form. Each stimulus could have one of two values on each dimension; thus, similarity was based upon the number of dimensions on which the stimuli were identical. In the case of perceptual comparisons, the two designs to be compared were presented physically for the subject to examine. In the case of the corresponding conceptual task, well-learned verbal labels for the two comparison stimuli were presented. It stands to reason, of course, that conceptual comparisons take longer to make, given the same level of accuracy. For one thing, conceptual tasks may require the search and retrieval (and/or activation) of memory representations of the two stimuli associated with the presented labels. But, in addition to search/retrieval (activation) time, it may also take longer to make a comparison in memory than it does to make a comparison between physically presented objects. Thus, there may be two components operating in the conceptual tasks, above and beyond those operating in the perceptual version: (1) search/retrieval (activation) of memory representations and (2) comparison of activated representations.

On the basis of available data, we expect RT in the conceptual task to depend on stimulus similarity, although the focus of this discrepancy is not clear. Similarity is likely to exert some influence on the time it takes to search and retrieve or activate the two representations in long-term memory on each trial. That is, two stimuli at disparate locations in long-term memory are likely to take longer to search out than two adjacent stimuli, on a simple spreadingactivation argument (Collins \& Loftus, 1975). If retrieved items are moved to short-term memory for side-by-side representation, as multistore models of memory (e.g., Atkinson \& Shiffrin, 1968) might be read to imply, comparison time should be unaffected by the distance between items in long-term memory (similarity). If, however, we assume an activation model of memory (e.g., Raaijmakers \& Shiffrin, 1981), similarity should manifest itself in the comparison operation. That is, two stimuli at disparate (activated) locations should take more time to compare than adjacent stimuli. Thus, although both models expect both slow and similaritydependent RTs in the conceptual task, the locus of the effect differs.

Consider "same" responses in the conceptual comparison task. The more dimensions of difference between two comparison stimuli, the slower the subject will be to identify the stimuli as "same" on the designated dimension. Response competition, distraction, and memorial processes (search/ retrieval, activation, or comparison) operate in concert to slow down RT. Expectations with regard to "different" responses are not quite so clear because of the possible offsetting effects of competition and distraction. With similarity-dependent memory processes, however, we should observe positive effects of similarity even for "different" responses in the conceptual task (which of course contrasts sharply with expectations in the perceptual task).

The present experiments develop a methodology to examine these issues. The methodology derives from a simple stagewise descriptive model of per- 
ceptual $\left(R_{\mathrm{p}}\right)$ and conceptual $\left(\mathrm{RT}_{\mathrm{c}}\right)$ comparison times, a model that includes, in addition to a constant base RT (B), contributions from previously identified response competition (C) and distraction (D) processes, both of which are similarity dependent. The most general statement of this model incorporates both similarity-independent $(\mu)$ and similarity-dependent (M) memory parameters for the conceptual task. The data, of course, will speak to the necessity of both terms. Thus,

$$
\begin{gathered}
\mathrm{RT}_{\mathrm{p}}=\mathrm{B}+\mathrm{c}(\mathrm{S})+\mathrm{d}(\mathrm{S}) \\
\mathrm{RT}_{\mathrm{c}}=\mathrm{B}+\mu+\mathrm{c}(\mathrm{S})+\mathrm{d}(\mathrm{S})+\mathrm{m}(\mathrm{S}),
\end{gathered}
$$

where $c(S), d(S)$, and $m(S)$ are similarity functions of the form $c(S)=C \cdot S ; C, D$, and $M$ in these functions are coefficients of response competition, distraction, and memory processes (undefined), respectively; and B and $\mu$ are constants for base RT and memory (undefined), respectively.

Experiment 1 examined the following set of expectations. In the case of perceptual comparisons, "same" judgments will be facilitated by increasing similarity; "different" judgments will exhibit less of an effect, being inhibited by similarity if response competition is stronger than distraction and facilitated if distraction is stronger than response competition. Conceptual comparisons will be slower because of the addition of the assumed memory processes. Under these conditions, both "same" and "different" responses are likely to be enhanced by increasing similarity, due to the greater adjacency of representation of similar items than of dissimilar items in memory. In Experiment 2, we attempted to determine the dependency of the memorial comparison process on similarity, exclusive of search/ retrieval (activation), by a procedure that allowed the subject to retrieve representations in advance of the comparison process. The results should tell us whether comparison per se, exclusive of retrieval, depends at all on the similarity of the two stimuli to be compared.

\section{EXPERIMENT 1}

\section{Method}

Subjects. The subjects were 60 undergraduate introductory psychology students who participated in partial fulfillment of a course requirement. The subjects served in single sessions, lasting $1.5 \mathrm{~h}$ each.

Design. The design included four within-subject variables: type of task (conceptual, perceptual comparisons), relevant dimension (size, color, shape), response ("same", "different"), and similarity (zero, one, two). Similarity was calibrated in terms of the number of dimensions, other than the relevant dimension, on which the two comparison stimuli were identical.

Trials were organized into six blocks, with 2 -min rests between blocks. The six blocks each consisted of 69 trials of either the conceptual or the perceptual task, with size, color, or shape selected as the relevant dimension. The first 5 trials of each block were practice trials. Of the remaining 64 trials, 32 were "same" response trials and 32 were "different" trials. Of each set of 32,8 trials had Similarity 0 (that is, the stimuli were different on both irrelevant dimensions), 16 trials had Similarity 1 ( 8 trials with each irrelevant dimension different), and 8 trials involved Similarity 2 .

Stimuli and Equipment. There were two types of stimulus slides employed in this study: geometric and syllabic. The geometric stimuli consisted of slides of geometric figures that varied along three dimensions-size, color, and shape-with each dimension taking one of two values, resulting in eight different figures. The values of color were yellow and black, of shape, triangle and square, and of size, large $(9 \mathrm{~mm}$ along the base) and small ( $3 \mathrm{~mm}$ along the base). The syllabic stimuli serving as names for geometric designs were taken from Noble's (1961) list of 2,100 CVC combinations. They consisted of $8 \mathrm{CVCs}$ that had both low meaningfulness and low associative values $\left(\mathrm{m}^{\prime}<\right.$ $\left.1.75, a^{\prime}<1.85\right)$.

The apparatus consisted of two Kodak Carousel slide projectors, with external Lafayette shutters, positioned side by side. The slide images were projected in pairs on a rear-projection screen $102 \mathrm{~cm}$ from the projector and $210 \mathrm{~cm}$ from the subjects, with the slide image areas coterminous. The subjects responded on a two-button box, with one button labeled "Same" and the other "Different." The labels were switched between subjects sc that half the subjects used the preferred hand for the "same" responses and half used the nonpreferred hand.

Procedure. The subjects first heard a set of instructions informing them of the nature of the task they were to perform. They were then given a set of eight cards, with each card bearing one of the eight geometrical stimuli on one side and a corresponding nonsense syllable label on the other side. The subjects studied the syllable-design correspondences on these cards freely for $15 \mathrm{~min}$ and were then tested, with feedback, over each of the pairs. This testing was performed by presenting the geometric stimuli and requiring the subjects to give the corresponding syllables, and vice versa. The subjects were required to give the eight correspondences between syllables and geometric stimuli correctly, in both directions, three consecutive times before they were allowed to continue with the experiment.

Prior to each block of trials, one stimulus dimension was designated as relevant. Then on each trial of the perceptual task, the subjects were presented with a pair of geometric stimuli. The subjects' task was to decide, while ignoring any differences on the other dimensions, whether the two figures were the same on the relevant dimension. In the conceptual task, the subjects were presented with a pair of nonsense syllables on each trial. In this task they were required to decode the syllables into the appropriate geometric figures according to the correspondences they had previously memorized, and then to decide whether the figures were the same on the designated relevant dimension. The same dimension was relevant for all trials within a block, but the relevant dimension changed between blocks. The subjects were instructed to keep their index fingers over the two buttons on the response box at all times so as to respond as quickly and as accurately as possible.

\section{Results}

Differences obtained largely, although not exclusively, in speed of response. Response accuracy, measured in proportion-correct responses, was relatively constant over conditions in this experiment. In no case was a decrease in RT accompanied by a decrease in correct responses, which precluded the possibility of a speed-accuracy tradeoff. Therefore, the primary data to be reported in this sec- 
tion are RTs. Unless otherwise indicated, all ps $<.05$.

There were several significant effects, but the source of variance of greatest interest in the RT data is the task (conceptual vs. perceptual) $\times$ response ("same" vs. "different") $\times$ similarity interaction, which was highly reliable $[F(2,118)=$ 161.08 , MSe $=.10]$. This interaction is shown in Table 1. Table 2 shows the corresponding error rates for each of the cells of this interaction. "Same" RTs decreased as similarity increased, the effect being numerically larger in the case of conceptual comparisons. In contrast, "different" RTs diverged in the conceptual and perceptual tasks with increases in similarity, becoming faster in the conceptual task and slower in the perceptual task.

It is clear that much of the effect captured in this triple interaction is attributable to the rapid "same" responses made to high similarity (identical) stimuli in the conceptual condition. Therefore, the data were reanalyzed, eliminating the Similarity 2 condition. The interaction of task $\times$ response $\times$ similarity was still reliable $[F(1,118)=74.75]$, although reduced in magnitude. On pairwise comparison, all three similarity conditions are significantly different in mean "different" RTs to conceptual stimuli. In the perceptual task, Similarity 0 differs reliably from Similarity 1 and Similarity 2 , but the latter two are statistically the same. All three similarity conditions differ significantly in mean "same" RTs to conceptual stimuli. Similarity 0 does not differ from Similarity 1 for perceptual stimuli, although both give reliably slower times than Similarity 2. Given this pattern of results, we feel secure in the conclusion that similarity affects "same" and "different" RTs differentially in conceptual and perceptual tasks.

Several other significant results were qualified by this interaction. The conceptual task produced significantly slower RTs than did the perceptual task $[F(1,59)=589.20, \mathrm{MSe}=2.92]$, the overall

Table 1

Reaction Times (in Milliseconds) for Correct Responses

\begin{tabular}{|c|c|c|c|c|c|c|}
\hline \multirow[b]{3}{*}{ Similarity } & \multicolumn{6}{|c|}{ Response } \\
\hline & \multicolumn{3}{|c|}{ Different } & \multicolumn{3}{|c|}{ Same } \\
\hline & 0 & 1 & 2 & 0 & 1 & 2 \\
\hline \multicolumn{7}{|c|}{ Experiment 1} \\
\hline $\begin{array}{l}\text { Perceptual } \\
\text { Concep tual }\end{array}$ & $\begin{array}{r}908 \\
2951\end{array}$ & $\begin{array}{r}933 \\
2909\end{array}$ & $\begin{array}{r}929 \\
2818\end{array}$ & $\begin{array}{r}913 \\
2945\end{array}$ & $\begin{array}{r}908 \\
2846\end{array}$ & $\begin{array}{r}864 \\
1693\end{array}$ \\
\hline \multicolumn{7}{|c|}{ Experiment $2-$ Normal } \\
\hline $\begin{array}{l}\text { Perceptual } \\
\text { Conceptual }\end{array}$ & $\begin{array}{r}839 \\
2477\end{array}$ & $\begin{array}{r}865 \\
2415\end{array}$ & $\begin{array}{r}902 \\
2368\end{array}$ & $\begin{array}{r}890 \\
2454\end{array}$ & $\begin{array}{r}860 \\
2378\end{array}$ & $\begin{array}{r}785 \\
1110\end{array}$ \\
\hline \multicolumn{7}{|c|}{ Experiment 2-Reverse Prime } \\
\hline $\begin{array}{l}\text { Perceptual } \\
\text { Conceptual }\end{array}$ & $\begin{array}{r}783 \\
2116\end{array}$ & $\begin{array}{r}955 \\
2100\end{array}$ & $\begin{array}{r}953 \\
1972\end{array}$ & $\begin{array}{r}906 \\
2081\end{array}$ & $\begin{array}{r}901 \\
2084\end{array}$ & $\begin{array}{l}487 \\
550\end{array}$ \\
\hline
\end{tabular}

Table 2

Error Rate (in Percent)

\begin{tabular}{lcccccc}
\hline & \multicolumn{7}{c}{ Response } \\
\cline { 2 - 7 } & \multicolumn{7}{c}{ Different } & \multicolumn{3}{c}{ Same } \\
Similarity & 0 & 1 & 2 & 0 & 1 & 2 \\
\hline \multicolumn{7}{c}{ Experiment 1} \\
Perceptual & 1.80 & 3.50 & 2.10 & 4.10 & 3.30 & 2.20 \\
Conceptual & 8.50 & 6.10 & 5.20 & 11.00 & 12.00 & 1.10 \\
& \multicolumn{7}{c}{ Experiment 2-Normal } \\
Perceptual & 2.31 & 2.27 & 2.78 & 4.93 & 2.88 & 1.90 \\
Conceptual & 9.47 & 7.67 & 7.10 & 10.13 & 9.67 & 1.23 \\
& Experiment & $2-$ Reverse Primed \\
Perceptual & 1.18 & 1.50 & 2.83 & 2.67 & 4.31 & .47 \\
Conceptual & 8.02 & 6.28 & 4.53 & 9.83 & 10.90 & .42 \\
\hline
\end{tabular}

difference being approximately $1.8 \mathrm{sec}$. There was a corresponding difference in error rates $(2.7 \%$ perceptual vs. $7.9 \%$ conceptual). The interactions of task $\times$ similarity and response $\times$ similarity were significant but of no consequence in view of the higher order interaction of these three variables. Relevant dimensions differed in RT, with responses to color being fastest and to shape slowest $[\mathrm{F}(2,118)$ $=11.15, \mathrm{MSe}=0.73$.

There was one curious and inexplicable interaction, relevant dimension $\times$ response $\times$ similarity $[\mathrm{F}(4,236)=9.29$, MSe $=.06]$. "Different" RTs increased with similarity when shape was the relevant dimension, whereas they decreased with similarity for the other two relevant dimensions. "Same" RTs decreased with increasing similarity in all cases, but the effect was slightly greater for shape than for the other two dimensions. Dimensional peculiarities are not unknown in geometric stimuli, and it is to this factor that we attribute the results (Gottwald \& Garner, 1975). This observation does not appear to compromise any of the major effects.

\section{Discussion}

The results obtained in the perceptual task are essentially the same as those reported earlier in the literature. "Same" reactions became faster and "different" reactions became slightly slower with increases in similarity. If we assume that response competition is stronger overall in its control of RT than is distraction, the results can be accounted for in terms of distraction and competition processes.

There was a huge scalar difference between RTs in the perceptual and the conceptual task. In addition, it was observed that both "same" and "different" RTs decreased with similarity in conceptual comparisons. If we assume the same two processes, response competition and distraction, are operative 
in basically the same way and magnitude in the conceptual task, how then does one account for the different functions observed in the conceptual and the perceptual task? First of all, there is a memory search, retrieval, or activation component involved in the conceptual task that is not present in the perceptual task. This component may be essentially automatic, but is nonetheless time consuming. If it is similarity dependent, then it could account both for the large scalar difference in RTs between the two comparison tasks and for the fact that their "different" RT functions are not parallel. But, as noted earlier, there is an additional process, the act of making comparisons in memory, the duration of which might depend on similarity. The argument is that comparison of items from adjacent activated representational points (similar items) is more rapid than comparison of items at distant locations (dissimilar items). If comparisons are made between activated nodes in long-term memory, then similarity should facilitate both "same" and "different" judgments in a conceptual task, consistent with the empirical functions observed in Experiment 1.

But this argument is based largely on conjecture. Because there are at least two factors, activation (search and/or retrieval) and memory comparison, that might operate in the conceptual task above and beyond those operating in the perceptual task, we really cannot be sure which of the two (or both) is similarity dependent. What we need is a method to eliminate either the activation (search and/or retrieval) or the comparison process. Experiment 2 addressed this issue.

\section{EXPERIMENT 2}

As previously discussed, one possible major difference between a multistore model of memory and an activation model lies in the expected location of the similarity effect in memory. A multistore model suggests that the locus of any similarity effect lies in search and retrieval, whereas an activation theory implies an effect in the memorial comparison operation. To establish the independence of search/retrieval and memorial comparison processes and to determine which is similarity dependent, we need a method for eliminating one process while still requiring the other. If such a procedure can be found and if conceptual-task RTs continue to depend, as they did in Experiment 1, on the similarity between comparison stimuli, then, at the very least, we would establish that the remaining process is similarity dependent.

The procedure used for this purpose involved a simple rearrangement of events in the conceptual task of Experiment 1. This rearrangement, which we refer to as reverse prime (RP), presented the subject with the two items for comparison in advance of naming the dimension on which they were to be compared. Thus, in the conceptual task, the subjects in Condition RP first saw two labels for the geometrical design to be compared. After ample time had been allowed for activating the representation corresponding to those labels, the dimension for comparison was identified and RT was measured from the onset of that signal. We argue that this RT is independent of time for activation (retrieval/search) and reflects primarily the additional operation of memory comparison above and beyond processes operative in the perceptual comparison task. A similar argument and procedure was used by Glass and Meany (1978) to show that item typicality affects the semantic categorization process, independently of its effect on activation or retrieval of item representation. The purpose of Experiment 2, then, was to compare performance in both conceptual and perceptual comparison tasks under the condition used in Experiment 1, henceforth called the normal condition, or Condition $\mathbf{N}$, with performance on the same tasks under Condition RP.

\section{Method}

Subjects. The subjects were 36 introductory psychology students who participated for 3 consecutive days, for $1.5 \mathrm{~h}$ on the 1st day and for $45 \mathrm{~min}$ on the 2 nd and 3rd days, in partial fulfillment of a course requirement.

Design. The design included six within-subject variables: days, task (conceptual, perceptual), prime condition (normal, reverse prime), relevant dimension (size, color, shape), response ("same," "different"), and similarity $(0,1,2)$. On each of 3 days there were 72 different types of trials, corresponding to the combination of these variables $(2 \times 2 \times 3 \times 2 \times 3)$. Each type of trial occurred four times on each day, resulting in 12 observations per subject per cell when collapsed over days. The factor of days was used simply as a replication and to examine possible practice effects. There were four main trialtype configurations of interest created by the interaction of task with prime condition: perceptual-normal, perceptual-reverse prime, conceptual-normal, and conceptual-reverse prime. Trials were run in blocks for each of these four trial-type configurations, but were randomized within each block with respect to the variables of relevant dimension, response, and similarity.

Stimuli and Apparatus. The stimuli were identical to those of Experiment 1, except that eight new CVC combinations were used. The CVCs used were again chosen for low meaningfulness (scaled meaningfulness, $\mathrm{m}^{\prime}<1.45$ ) and low associative value (rated associations, $a^{\prime}<1.60$ ).

Except for the addition of a third projector to show the priming slide (naming the relevant dimension), the apparatus operated in the same way as in Experiment 1 . The centers of the slide images projected on the rear viewing screen formed an equilateral triangle, with the edges of the slide images coterminous. On normal trials, the upper projector opened first, to present the prime (size, color, or shape) slide. After a $1.5-\mathrm{sec}$ delay, the shutters on the two lower projectors opened to reveal the pair of stimulus items. In reverse-prime mode, the order of presentation was reversed: stimuli first and prime $1.5 \mathrm{sec}$ later. The opening of the final shutter(s) caused a timer to be activated. The prime interval of $1.5 \mathrm{sec}$ had been determined on the basis of pilot data with practiced subjects to be adequate to allow 
for decoding of the syllable stimuli to their geometric referents. Procedure. On the 1st day, the subjects were familiarized with the stimulus materials and tested on the correspondences as in Experiment 1. The subjects were then instructed about the nature of the task and presented practice trials, as in Experiment 1 . After these trials, the subjects were informed about the difference between normal and reverse-prime trials. On normal trials, they were to note the contents of the first slide and, when the stimulus slides appeared $1.5 \mathrm{sec}$ later, to make a similarity judgment based on the previously named dimension. On reverse-prime trials, the subjects were instructed to use the 1.5-sec interval to "decode" the labels. Following these additional instructions, the subjects were each given 20 more practice trials with feedback. Half of these trials used geometric stimuli, and half used nonsense syllables. Of each of these trial types, half were normal and half were reverse prime. The order of trials was random.

In the experiment proper, the subjects were presented with 78 trials of each of the four types, presented in blocks, with a 3-min pause between blocks. The ordering of the blocks was counterbalanced across days and across subjects such that the same sequence of blocks was never presented to a subject on more than 1 day, and each type of block was represented equally often in each serial position.

On the 2 nd and 3 rd days of the experiment, the subjects were first given $10 \mathrm{~min}$ to restudy the geometric figures, nonsense syllables, and their correspondences. Following this, they were again tested on the material in a manner identical to that on the 1st day.

\section{Results and Discussion}

As in Experiment 1, major effects are evidenced primarily in the speed rather than the accuracy measure, again, with no evidence of a speed-accuracy tradeoff. Primary interest lies in two interactions, task $\times$ response $\times$ similarity, the interaction of significance in Experiment 1, and task $\times$ response $x$ similarity $X$ prime condition.

If practice and prime condition have no interactive effects, we would expect the first interaction to parallel the outcome of Experiment 1. Significance of the second interaction would tell us something about the role of activation (search/retrieval) vs. comparison processes in the memorial task. From Experiment 1, we know that the similarity functions for "different" responses are not the same in conceptual and perceptual tasks under Condition $\mathbf{N}$. The critical consideration in the present data is the similarity function that obtains for "different" responses in Condition RP. If providing the subject with labels for the two stimuli to be compared allows the subject not only to activate the corresponding nodes in memory but also to bring them forward for side-by-side comparison in working memory, then we would expect roughly the same similarity function for "different" responses under the conceptual task as under the perceptual task, even though considerably more time may be needed in the conceptual case. Thus, if Condition RP facilitates the comparison process by permitting anticipatory retrieval of nodes to be compared, we should get a reliable four-way interaction of task $\times$ response $\times$ similarity $\times$ prime con- dition. If, on the other hand, Condition RP facilitates node activation only (and not the comparison process), then there will not be an interaction. Facilitation of the activation process in Condition RP should make conceptual responses faster (and possibly more accurate). It should not change the nature of the similarity function for "different" (or "same") responses, however, because the distance between activated nodes is, by assumption, based on similarity.

The data, broken down according to the four major variables, are presented in Table 1 . The threeway interaction of task $\times$ response $\times$ similarity was highly significant $[\mathrm{F}(2,70)=94.04, \mathrm{MSe}=.25]$ in RTs, even when Similarity 2 data were eliminated $[F(1,70)=13.64]$. The four-way interaction was unreliable in both cases. As can be seen from Table 1 , the major influence of Condition RP was to decrease RTs by approximately $350 \mathrm{msec}$ across the board in the conceptual task. Perceptual comparison times showed little effect.

The RTs obtained in this experiment are, in general, slightly faster than those reported in Experiment 1 . This can be understood in terms of a significant main effect of practice (days) $[F(2,70)=$ 177.37, $\mathrm{MSe}=2.02$ ], reflecting the fact that $\mathrm{RT}$ decreases by an average of approximately $700 \mathrm{msec}$ over the 3 days. But, this practice effect interacts with none of the other major variables of the experiment. It should also be noted that the dimension used as the basis of comparison produced a reliable difference in $R T[F(2,70)=41.57$, MSe $=$ .77] and errors. Overall, the subjects were quicker to respond when the task was to judge stimuli on the basis of size or color than when a shape judgment was required. Relevant dimension interacted with similarity, response, and type of task, although these effects do nothing to compromise the basic major conclusion about the influence of prime condition on conceptual and perceptual comparisons.

\section{GENERAL DISCUSSION}

The results of these two experiments portray a clear picture of the differences between perceptual and conceptual comparisons of formally identical stimuli and of the effects of stimulus similarity on the relevant subprocesses. First, consider perceptual comparisons. Using an existing large data base, we argued that stimulus similarity, calibrated in terms of the number of overlapping features on independent stimulus dimensions, would affect performance through two mechanisms. A distraction process should slow both "same" and "different" responses. Response competition, in contrast, should have a differential effect on "same" and "different" responses. As similarity increases, "same" responses should be speeded and "differ- 
ent" responses should be slowed. These arguments lead to a simple linear model portrayed in Equations 1 and 2.

The outcomes of both experiments are consistent with this model if one assumes that the response competition process is stronger than the distraction process. In fact, the data suggest that distraction, if it operated at all in these data, operated minimally. A simpler model that ignores the distraction parameter altogether is just as consistent, quantitatively, with the outcome of both experiments as is the more complex two-parameter model. For purposes of this analysis, data for "same" responses with $S=2$ were eliminated. These data are particularly troublesome in the conceptual RT case in which subjects are presented with two identical nonsense labels. No retrieval process need be involved to make the correct ("same") response. Thus, for purposes of these data we argue: $R_{\mathrm{p}}=$ $B+c(S)$, where $c(S)=C \cdot S$.

Data from conceptual problems are consistent with the simple model developed for perceptual comparisons as far as it goes. They require, however, minimally, one additional parameter. As we have noted, there are at least two ways in which memory might enter to affect RTs when the comparisons are indirect. First, it takes time for the presented stimuli (labels) either to retrieve or to activate the objects (representations) for memorial comparisons. Second, comparisons might be slower for stimuli in memory than for stimuli in the visual field. Any or all of the following processessearch and retrieval, activation, and memorial comparison-could, under certain models, be similarity dependent. One contribution of the present set of data is to elucidate the focus of the similarity effect.

Consider the following argument. Memorial comparisons occur in short-term memory. That is to say, for a comparison of this sort to take place, the subject must be aware of the two stimuli. If one adopts a theory of memory that assumes separate long-term and short-term stores, then similarity (if it is effective at all) is likely to affect retrieval from long-term to short-term memory. On a semantic network argument, retrieval to shortterm memory may be more rapid if the two stimuli in question have adjacent addresses in long-term memory than if they are more distantly placed. The comparison of stimuli, represented "side by side" in short-term memory, however, is not affected by similarity except for ways already considered in perceptual comparisons. Suppose, on the other hand, that short-term memory, rather than having a unique location, is considered to be the activated portion of long-term memory. There is no retrieval, then, of representations from long-term into shortterm store. Rather, it is a matter of "lighting up" the nodes corresponding to the two to-be-compared stimuli. Activation (the counterpart of search/ retrieval in the alternative model) might be similarity dependent in the same sense as search/retrieval. But in this case, for reasons of semantic distance, the comparison process itself is similarity dependent, because it should be easier to compare adjacent activated nodes than more distant nodes.

The data of Experiment 2 speak to the distinction between these two possibilities. Condition RP was designed to eliminate retrieval or activation processes from response times. In this case, nodes corresponding to the two comparison stimuli are activated in advance of knowing the basis (dimension) of comparison. RT is measured from the point of cuing the basis of comparison to the completion of the comparison process itself. The effect of similarity was virtually identical under Conditions $\mathbf{N}$ and RP, although obviously RTs were significantly lower by a constant $350 \mathrm{msec}$ for Condition RP. This implies that it is the comparison process per se, rather than (or in addition to) retrieval or activation that is similarity dependent. It further implies that an activation as opposed to a retrieval model of short-term memory is more appropriate for the data reported. ${ }^{1}$

Again, it is possible to write a relatively simple linear model for RTs in the conceptual task. What is required at a minimum, above and beyond the model for perceptual comparisons, are two additional components, one of which is and another which is not similarity dependent. Thus, $R T_{c}=$ $\mathbf{B}+\mu+\mathrm{c}(\mathbf{S})+\mathbf{m}(\mathbf{S})$, where $\mathbf{m}(\mathbf{S})=\mathbf{M} \cdot \mathbf{S}$.

To examine this model, a subset of the data obtained in Experiments 1 and 2 was used to provide parameter values for the model. The model, incorporating those values, was then used to "predict" the remainder of the data set. The values used in the model are shown in Table 3. These values were obtained from the experimental data for the "different" response condition, with similarity values 0 and 1. A comparison of the obtained and predicted data for Experiments 1 and 2 is given in Table 4 . The correlations between obtained and predicted data for the perceptual and conceptual conditions, respectively, were $r=.995$ and $r=.955$ for Experiment 1 and $r=.908$ and $r=.999$ for Experiment 2.

Table 3

Model Parameter Estimates (in Milliseconds)

\begin{tabular}{crc}
\hline & Experiment 1 & Experiment 2 \\
\hline B & 908 & 839 \\
$\mu$ & 2042 & 1638 \\
C & 25 & 26 \\
M & 67 & 88 \\
\hline
\end{tabular}


Table 4

Obtained and Model-Generated Reaction Time Values

\begin{tabular}{|c|c|c|c|c|c|c|}
\hline \multirow[b]{3}{*}{ Similarity } & & \multicolumn{5}{|c|}{ Response } \\
\hline & & \multicolumn{3}{|c|}{ Different } & \multicolumn{2}{|c|}{ Same } \\
\hline & & 0 & 1 & 2 & 0 & 1 \\
\hline \multicolumn{7}{|c|}{ Experiment 1} \\
\hline Perceptual & $\begin{array}{l}\text { Obtained } \\
\text { Model }\end{array}$ & $908^{*}$ & $933^{*}$ & $\begin{array}{l}929 \\
958\end{array}$ & $\begin{array}{l}913 \\
908\end{array}$ & $\begin{array}{l}908 \\
883\end{array}$ \\
\hline Conceptual & $\begin{array}{l}\text { Obtained } \\
\text { Model }\end{array}$ & $2951 *$ & $2909^{*}$ & $\begin{array}{l}2818 \\
2867\end{array}$ & $\begin{array}{l}2945 \\
2950\end{array}$ & $\begin{array}{l}2846 \\
2858\end{array}$ \\
\hline \multicolumn{7}{|c|}{ Experiment 2-Normal } \\
\hline Perceptual & $\begin{array}{l}\text { Obtained } \\
\text { Model }\end{array}$ & $839^{*}$ & $865^{*}$ & $\begin{array}{l}902 \\
891\end{array}$ & $\begin{array}{l}890 \\
839\end{array}$ & $\begin{array}{l}860 \\
813\end{array}$ \\
\hline Conceptual & $\begin{array}{l}\text { Obtained } \\
\text { Model }\end{array}$ & $2477^{*}$ & $2415^{*}$ & $\begin{array}{l}2368 \\
2353\end{array}$ & $\begin{array}{l}2454 \\
2477\end{array}$ & $\begin{array}{l}2378 \\
2363\end{array}$ \\
\hline \multicolumn{7}{|c|}{ Experiment 2-Reverse Prime } \\
\hline Perceptual & $\begin{array}{l}\text { Obtained } \\
\text { Model }\end{array}$ & $\begin{array}{l}783 \\
839\end{array}$ & $\begin{array}{l}955 \\
865\end{array}$ & $\begin{array}{l}953 \\
891\end{array}$ & $\begin{array}{l}906 \\
839\end{array}$ & $\begin{array}{l}901 \\
813\end{array}$ \\
\hline Conceptual & $\begin{array}{l}\text { Obtained } \\
\text { Model }\end{array}$ & $\begin{array}{l}2116 \\
2129\end{array}$ & $\begin{array}{l}2100 \\
2067\end{array}$ & $\begin{array}{l}1972 \\
2005\end{array}$ & $\begin{array}{l}2081 \\
2129\end{array}$ & $\begin{array}{l}2084 \\
2015\end{array}$ \\
\hline
\end{tabular}

*Values used to estimate parameters.

As can be seen from Table 3, there is a great deal of consistency across experiments for the values of $C$, the response bias effect, and $M$, the effect of similarity on memory. Indeed, simple descriptive models that incorporate only two similaritydependent parameters, response competition and memorial comparison, appear to account in reasonable detail for the general results of these two experiments.

\section{REFERENCES}

Atkinson, R. C., \& Shiffrin, R. M. Human memory: A proposed system and its control processes. In K. W. Spence \& J. T. Spence (Eds.), The psychology of learning and motivation: Advances in research and theory (Vol. 2). New York: Academic Press, 1968.

Caramazza, A., Hersh, H., \& Torgenson, W. Subjective structures and operations in semantic memory. Journal of Verbal Learning and Verbal Memory, 1976, 15, 103-117.

Collıns, A. M., \& Loftus, E. F. A spreading-activation theory of semantic processing. Psychological Review, 1975, 82, 407-428.

Connor, J. M. Effects of increased processing load on parallel processing of visual displays. Perception \& Psychophysics, $1972,12,121-128$.

Egeth, H. E. Parallel versus serial processes in multidimensional stimulus discrimination. Perception \& Psychophysics, 1966, 1, 245-251.

Glass, A., \& MEANy, P. Evidence for two kinds of low-typical instances in a categorization task. Memory \& Cognition, 1978, 6, 622-628.

GotTwald, R. L., \& Garner, W. R. Filtering and condensation tasks with integral and separable dimensions. Perception \& Psychophysics, 1975, 18, 26-28.

Hawkins, H. L., \& Shigley, R. H. Irrelevant information and processing mode in speeded discrimination. Journal of Experimental Psychology, 1972, 96, 389-395.

Kosslyn, S., Murphy, G., Bemesderfer, M., \& Feinstein, K. Category and continuums in mental comparisons. Journal of Experimental Psychology: General, 1977, 106, 341-375.
KRUEGER, L. E. Effects of irrelevant surrounding material on speed of same-different judgment of two adjacent letters. Journal of Experimental Psychology, 1973, 98, 252-259.

Miller, J., \& Bauer, D. Irrelevant differences in the "same""different" task. Journal of Experimental Psychology: Human Perception and Performance, 1981, 7, 196-207.

Moyer, R. S., \& BAyer, R. H. Mental comparisons and the symbolic distance effect. Cognitive Psychology, 1976, 8, 228-246.

NoBle, C. E. Measurements of association value (a), rated associations $\left(a^{\prime}\right)$, and scaled meaningfulness $\left(m^{\prime}\right)$ for the 2100 CVC combinations of the English alphabet. Psychological Reports, 1961, 8, 487-521.

RaAijmakers, J. G. W., \& Shiffrin, R. M. Search of associative memory. Psychological Review, 1981, 88, 93-134.

TVersky, B. Pictorial and verbal encoding in a short-term memory task. Perception \& Psychophysics, 1969, 6, 225-233.

Well, A. D., \& Green, J. Effects of color differences in a letter matching task. Psychonomic Science, 1972, 29, 109-110.

Williams, C. The effect of an irrelevant dimension on "samedifferent" judgments of multi-dimensional stimuli. Quarterly Journal of Experimental Psychology, 1974, 26, 26-31.

\section{NOTE}

1. One possible counterargument would be that despite what our pilot work had indicated, the 1.5-sec priming time that was allowed for subjects to decode the conceptual stimuli into their perceptual referents was not adequate. Evidence that this was not the case comes from a comparison of the data of the normal conditions for Days 2 and 3. The best estimate of the time required to decode the conceptual stimuli is provided by the difference between the perceptual and conceptual conditions in which the RT clock started when the stimuli were presented (the normal conditions). For these days, the difference between the perceptual-normal and the conceptual-normal is less than $1.5 \mathrm{sec}(1.196 \mathrm{sec}$ for Day 2 and $0.926 \mathrm{sec}$ for Day 3). The fact that there was no interaction in the effect of similarity between Conditions $\mathbf{N}$ and RP for either of these days suggests that the above argument against our results is not valid. revision accepted for publication January $11,1983$. ) 